\title{
An estimation of the p-adic sizes of common zeros of partial derivative polynomials of degree six
}

\begin{abstract}

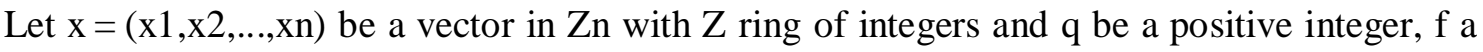
polynomial in $\mathrm{x}$ with coefficient in $\mathrm{Z}$. The exponential sum associated with $\mathrm{f}$ is defined as $\mathrm{S}(\mathrm{f} ; \mathrm{q})$ 田 $\mathbb{X}$ xmodqe $\left(\left(2^{\prime}\right.\right.$ if $\left.\left.(\mathrm{x})\right) / \mathrm{q}\right)$ where the sum is taken over a complete set of residues modulo q. The value of S (f; q) depends on the estimate of cardinality $|\mathrm{V}|$, the number of elements contained in the set $\mathrm{V}=\{\mathrm{xmodq} \mid \mathrm{fx} f 0$ modq $\}$ where $\mathrm{fx}$ is the partial derivatives of $\mathrm{f}$ with respect to $\mathrm{x}$. To determine the cardinality of $\mathrm{V}$, the $\mathrm{p}$-adic sizes of common zeros of the partial derivative polynomials need to be obtained. In this paper, we estimate the p-adic sizes of common zeros of partial derivative polynomials of $f(x, y)$ in $\mathrm{Zp}[\mathrm{x}, \mathrm{y}]$ with a sixth degree form by using Newton polyhedron technique. The polynomial is of the form $f(x, y) \llbracket \mathbb{a l l} 66+b x 5 y+c x 4 y 2+s x+t y+k$.
\end{abstract}

Keyword: Cardinality; Exponential sums; Newton polyhedron; p-adic sizes 